73rd Conference of the Italian Thermal Machines Engineering Association (ATI 2018), 12-14 September 2018, Pisa, Italy

\title{
Development of an on-line energy management strategy for hybrid electric vehicle
}

\author{
Tufano Daniela ${ }^{a^{*}}$, De Bellis Vincenzo ${ }^{a}$, Malfi Enrica ${ }^{a}$ \\ ${ }^{a}$ Industrial Engineering Department, University of Naples "Federico II", Via Claudio 21, 80125 Naples, Italy
}

\begin{abstract}
The Hybrid Electric Vehicle (HEV) seems to be one of the most promising short-term solution to improve the sustainability on the transportation sector. As well-known, the numerical analyses can give a substantial contribute during the preliminary vehicle design. In this context, the development of the Energy Management Strategy (EMS) represents the most challenging task. In this paper, an on-line local optimization EMS for a parallel/series hybrid vehicle is proposed to minimize the $\mathrm{CO}_{2}$ emissions. The proposed EMS, implemented in a dynamic simulation platform, is compared to the well-assessed off-line Pontryagin's Minimum Principle (PMP). Firstly, the main differences regarding the energy management are highlighted in detail. Then, the EMSs are assessed in terms of $\mathrm{CO}_{2}$ emissions, putting into evidence that the proposed on-line strategy involves limited penalizations (3-4\%) compared to the PMP target.
\end{abstract}

(C) 2018 The Authors. Published by Elsevier Ltd.

This is an open access article under the CC BY-NC-ND license (https://creativecommons.org/licenses/by-nc-nd/4.0/)

Selection and peer-review under responsibility of the scientific committee of the 73rd Conference of the Italian Thermal Machines Engineering Association (ATI 2018).

Keywords: Hybrid electric vehcile; vehicle simulation, energy management strategy, local optimization strategy

\footnotetext{
* Corresponding author. Tel.: +39081-7683293; fax: +39 0812394165 .
}

E-mail address: daniela.tufano@unina.it

\section{Acronyms}

BSFC Brake Specific Fuel Consumption

EFR Equivalent Fuel Rate

EFRMS Equivalent Fuel Rate Minimization Strategy 


\begin{tabular}{|ll} 
GOS & Global Optimization Strategy \\
HEV & Hybrid Electric Vehicle \\
ICE & Internal Combustion Engine \\
LOS & Local Optimization Strategy \\
MGU & Motor Generator Unit \\
PMP & Pontryagin's Minimum Principle \\
SOC & State Of Charge \\
WLTC & Worldwide harmonized Light vehicles Test Cycle \\
Symbols & \\
$P$ & Power \\
$t$ & Current time along the driving cycle \\
$T_{\text {WLTC }}$ & Total driving cycle time \\
Subscript & \\
req & Required
\end{tabular}

\section{Introduction}

Nowadays, Hybrid Electric Vehicles (HEVs) have undergone a considerable development since they have proved to be greatly effective in reducing the fuel consumption, along with the $\mathrm{CO}_{2}$ emissions, compared to conventional vehicles [1]. Although this kind of vehicles turns out to be highly advantageous, their architecture is more complex and articulated than the traditional ones.

Generally speaking, a hybrid powertrain includes a thermal unit (Internal Combustion Engine - ICE), which is coupled in series and/or parallel to one or more electric units, linked to an energy storage device, usually consisting of a battery [2]. The energy management on vehicle can be gained by switching among various operating modes, maximizing the fuel economy. This target has been achieved through several control strategies, which have been introduced in the last years. They are addressed to the minimization of a cost function, especially regarding the fuel consumption, while controlling the State Of Charge (SOC) of the battery. This could be considered as a constrained optimization problem. The task of the Energy Management Strategy (EMS) is to solve a minimization problem, subjected to system dynamics and constraints. For this purpose, various methods have been developed, providing different approaches to handle the energy flow in the hybrid electric vehicles. The EMSs can be classified as follows [3]: Heuristic Strategies [4], Global Optimization Strategies (GOS) and Local Optimization Strategies (LOS). The first and the last are on-line strategies, based only on present or past information. Contrarily the second ones require also future information, so they are commonly called off-line strategies. The GOS has the advantage, knowing ahead the entire driving cycle, of yielding the optimal solution for the control problem. At the same time, this entails its impossibility to be implemented on a real vehicle due to the missing knowledge of the future information. So, the GOS can be used only in simulations. The most recognized GOSs are the Pontryagin's Minimum Principle (PMP) [5] and the "dynamic programming" [6]. The GOSs are generally used as "theoretical targets" to find the optimal results yielded by the hybrid vehicle. About the LOS, it reduces the above-mentioned global problem in a succession of local problems not solved on the entire driving cycle. In this way, there is no need for future information, so that the strategy could be implemented on a real application. Of course, the solution provided by the LOS is not able to ensure the optimality of the control problem, providing hence a suboptimal result. The best-known LOSs are the "equivalent consumption minimization strategy" [7] and the "stochastic dynamic programming" [8]. The main aim of this work is the description of a novel LOS, not included in the above recalled categories. The proposed LOS is implemented in a simulation platform and applied to a reference C segment vehicle along a WLTC [9]. The effectiveness of the approach is verified against the PMP outcomes, highlighting the main differences in the management of the thermal and the electric units, and in terms of $\mathrm{CO}_{2}$ emissions. 


\section{Vehicle architecture and modelling}

A HEV of the segment $\mathrm{C}$ with parallel/series powertrain is investigated in this work. Its schematization and main features are shown in Fig. 1. The vehicle is equipped with an ICE, two electric Motor/Generator Units $\left(\mathrm{MGU}_{1-2}\right)$, one battery, three clutches $\left(\mathrm{Clutch}_{1-3}\right)$ and two gearboxes $\left(\right.$ Gearbox $\left._{1-2}\right)$. Two thermal units are considered, characterized by similar maps of Brake Specific Fuel Consumption (BSFC), but with different minimum levels (BSFC $\left.\mathrm{B}_{1-2}\right)$. Because of confidentiality reasons, the BSFC values are not shown in the text. The energy recovery, obtained by the regenerative braking, can be carried out by means of the $\mathrm{MGU}_{2}$. For the examined vehicle, two main modes are available, each of them involving a number of sub-modes. In the series one, the vehicle is moved only by the $\mathrm{MGU}_{2}$ and it is provided with three available sub-states. The first is the pure electric vehicle; in this, the electric unit $\mathrm{MGU}_{2}$ fully supplies the tractive demand, with the only clutch ${ }_{3}$ engaged. In the second, the charging mode $e_{1}$, the ICE charges the battery through the $\mathrm{MGU}_{1}$ with the clutch 1 engaged, while the $\mathrm{MGU}_{2}$ moves the vehicle. In the last, the $\mathrm{MGU}_{1}$ provides energy for the ICE take-off, with the clutch ${ }_{1}$ engaged. Concerning the parallel mode, the vehicle is driven by both ICE and $\mathrm{MGU}_{2}$ and also here three modalities are available. In the pure ICE mode, the only ICE drives the vehicle, while the $\mathrm{MGU}_{1}$ and the $\mathrm{MGU}_{2}$ are turned off. In the hybrid propulsion, the $\mathrm{MGU}_{2}$ supports the vehicle driving (power-split principle), when ICE is unable to fully supply the vehicle power demand. Finally, in the charging mode $_{2}$, the ICE supplies power to the battery and to the vehicle.

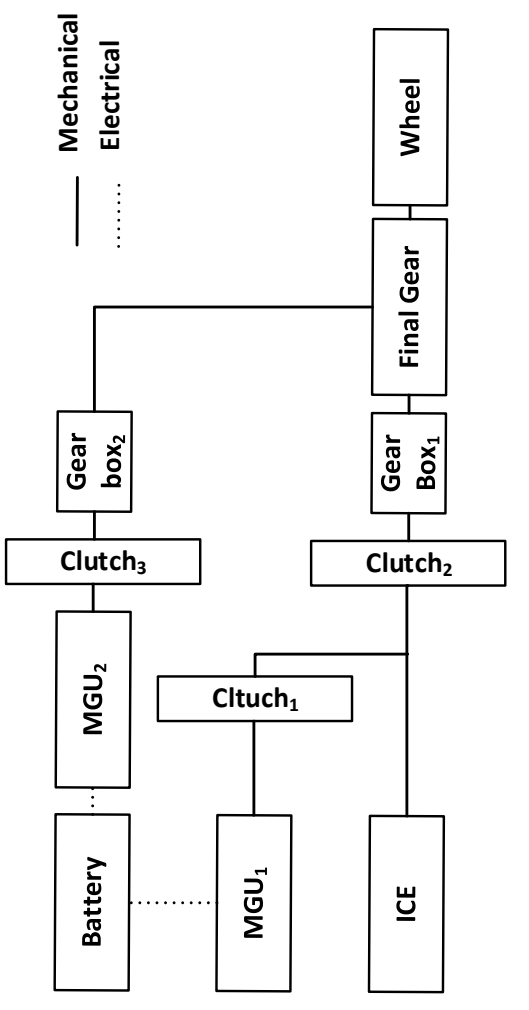

\begin{tabular}{|c|c|c|}
\hline & Vehicle Features & \\
\hline \multirow{3}{*}{$\begin{array}{c}\text { Internal Combustion } \\
\text { Engine }\end{array}$} & Displacement, $\mathrm{cm}^{3}$ & 1633.12 \\
\hline & Max Power, kW & 125 \\
\hline & Inertia, $\mathrm{kg}^{*} \mathrm{~m}^{2}$ & 0.35 \\
\hline \multirow{3}{*}{$\begin{array}{l}\text { Motor Generator Unit } \\
\qquad\left(\mathrm{MGU}_{1}\right)\end{array}$} & Max Power, kW & 55 \\
\hline & Max Torque, Nm & 165 \\
\hline & Inertia, $\mathrm{kg}^{*} \mathrm{~m}^{2}$ & 0.10 \\
\hline \multirow{3}{*}{$\begin{array}{l}\text { Motor Generator Unit } \\
\qquad\left(\mathrm{MGU}_{2}\right)\end{array}$} & Max Power, $\mathrm{kW}$ & 50 \\
\hline & Max Torque, Nm & 240 \\
\hline & Inertia, $\mathrm{kg}^{*} \mathrm{~m}^{2}$ & 0.10 \\
\hline \multirow{4}{*}{ Battery } & Internal Resistance, Ohm & 0.375 \\
\hline & Voltage, Volt & 400.0 \\
\hline & Energy density, Wh/kg & 170.00 \\
\hline & Usable battery sizing, $\mathrm{kWh}$ & 0.50 \\
\hline \multirow{5}{*}{ Vehicle } & Car aero drag, $\mathrm{m}^{2}$ & 0.775 \\
\hline & Tire rolling resistance, $\mathrm{kg} / \mathrm{t}$ & 8 \\
\hline & Wheel diameter, $\mathrm{m}$ & 0.723 \\
\hline & Axle ratio, - & 4.4 \\
\hline & Axle inertia, $\mathrm{kg}^{*} \mathrm{~m}^{2}$ & 1.5 \\
\hline \multirow{4}{*}{ Gear Box 1} & Gear 1 Ratio, - & 2.7 \\
\hline & Gear 2 Ratio, - & 1.6 \\
\hline & Gear 3 Ratio, - & 1.0 \\
\hline & Gear 4 Ratio, - & 0.6 \\
\hline \multirow{2}{*}{ Gear Box 2} & Gear 1 Ratio, - & 2.7 \\
\hline & Gear 2 Ratio, - & 1.0 \\
\hline
\end{tabular}

Fig. 1. Powertrain schematic (left). Main vehicle characteristics (right)

Regarding the vehicle simulation, each component of the schematic in Fig. 1 is described by a lumped-parameter model. The required tractive demand at the wheels considers the vehicle inertial forces and resistances (aerodynamic and rolling load). The thermal unit is characterized by a quasi-steady map-based approach. The maps of brake mean effective pressure, friction mean effective pressure and BSFC are implemented as a function of the engine speed and 
of the accelerator pedal position. $\mathrm{A} \mathrm{CO}_{2}$ map is not directly considered, and the vehicle $\mathrm{CO}_{2}$ emissions are derived by the fuel consumption along the cycle, expressed in $\mathrm{g}_{\text {fuel }} / \mathrm{km}$, through a constant conversion factor of $3.2 \mathrm{~g}_{\mathrm{co} 2} / \mathrm{km}$. This last is estimated for a stoichiometric combustion of a reference commercial oxygenated gasoline $\left(\mathrm{CH}_{1.92} \mathrm{O}_{0.03}\right)$. This simplified assumption seems reasonable taking into account that the considered ICE works with a stoichiometric airfuel ratio over the whole operating plane. For both MGUs, the maximum brake torque curve is implemented in the model, assuming a constant efficiency of 0.9025 . The battery is described by a conventional SOC model, which calculates the current SOC on the basis of the power absorbed from or supplied to the battery, depending on the electric flux [9]. An internal resistance is imposed to compute the Joule-effect losses. Finally, the mechanical losses in the gearbox are estimated assuming a constant efficiency of 0.97 .

\section{Energy Management Strategies}

In the present work, two different EMSs, addressed to the fuel consumption minimization, are analyzed: the wellknown PMP and a novel approach developed by the authors, labelled as Equivalent Fuel Rate Minimization Strategy (EFRMS). The PMP, thanks to the knowledge of the driving cycle, provides an optimal solution of the control problem, and it is generally used to assess the potential of a vehicle concept under development. Therefore, here it is used as a benchmark for the EFRMS. As explained in [5], the PMP is based on the minimization of the Hamiltonian function, under a number of constraints, also including the same initial and final SOC of the battery along the driving cycle.

Differing from the PMP, the EFRMS solves a local optimal problem. This means that, at each time, it selects the problem control variables with the aim of minimizing the instantaneous fuel rate, but not the "global" fuel consumption along the driving cycle. The developed logics choose the vehicle mode, between series and parallel, the power requests for the MGUs, the ICE and the brakes, the gear number and the clutch positions, as schematically represented in the flowchart in Fig. 2a.

The first choice of the EFRMS is between the series and parallel modalities. To this aim, two "equivalent" fuel rates $\left(E_{\mathrm{SFR}}\right)$ are calculated and compared: the fuel rate in series mode $\left(\mathrm{EFR}_{\text {series }}\right)$ and in parallel mode $\left(\mathrm{EFR}_{\text {parallel }}\right)$. Both are function of the power demand required by the vehicle and of the SOC. The concept of the series/parallel selection logic is summarized in the mathematical expression (1). To avoid excessive fluctuations between series/parallel modes and engine on/off, at each time step a tunable penalization factor, $\mathrm{c}_{t}$, enhances the EFR of the mode not used. This means that if the series (parallel) mode is active, $\mathrm{EFR}_{\text {parallel }}\left(\mathrm{EFR}_{\text {series }}\right)$ is multiplied by $\mathrm{c}_{\mathrm{t}}$.

$$
\forall t \in[0, T] \min E F R\left(P_{\text {req }}, S O C\right)\left\{\begin{array}{l}
E F R_{\text {series }}(t)>E F R_{\text {parallel }}(t) \Rightarrow \min E F R=E F R_{\text {parallel }}(t) \\
E_{\text {series }}(t)<E F R_{\text {parallel }}(t) \Rightarrow \min E F R=E F R_{\text {series }}(t)
\end{array}\right.
$$

The EFRMS includes a simple threshold-based strategy for the charging mode. This last is activated (deactivated) when the SOC reaches the lower (upper) threshold. The operating SOC band is set between 0.2 and 0.9 by a preliminary sensitivity analysis. This range proves to ensure an optimal employment of the battery capacity, avoiding a full charging/discharging. The EFRMS also entails a regenerative braking modality to charge the battery during the vehicle decelerations. The amount of recovered energy is limited by the current SOC level and by the breaking capacity of the $\mathrm{MGU}_{2}$.

The most crucial point of the proposed strategy is the estimation of the EFR in series and parallel modes. The logics of the EFR calculations are summarized in the flowcharts of Fig. 2b. Staring from the series mode, it is expected that the vehicle is mainly in a pure electric driving (charging mode disabled), so then no fuel is consumed by the ICE. For this reason, the control strategy can only estimate an "equivalent" fuel rate. The EFR series $_{\text {is calculated as the product }}$ of the minimum BSFC and of the power requested to the ICE. This last is computed starting from the vehicle power demand at the wheels and considering all the losses along the energy path (gearbox ${ }_{2}, \mathrm{MGU}_{2}$, battery and $\mathrm{MGU}_{1}$ ), following the flowchart in Fig. 2b). When the charging mode is activated, an additional fuel rate contribution is considered in the $\mathrm{EFR}_{\text {series }}$ calculation, corresponding to the quantity consumed by the ICE in the operating point of minimum BSFC. 
The fuel rate estimation in parallel mode is schematized in the right side of the flowchart of Fig. 2b. Differently from the series mode, the control logic additionally has to identify the optimal gearbox setting. The main steps of this task are summarized in Fig. 3. To this purpose, once fixed the current vehicle speed, the engine rotational speed is derived for each gear (Fig. 3a). Due to noise, vibration and harshness issues, the only gear ratios considered are the ones ensuring an ICE speed above a certain threshold level (the fourth gear ratio is discarded in the example of Fig. 3a). Then, for the remaining gear ratios, the engine maximum power is evaluated (Fig. 3b). The gear ratios, not ensuring the fulfilment of the power demand, are hence discarded (the third gear ratio in the example of Fig. 3b).
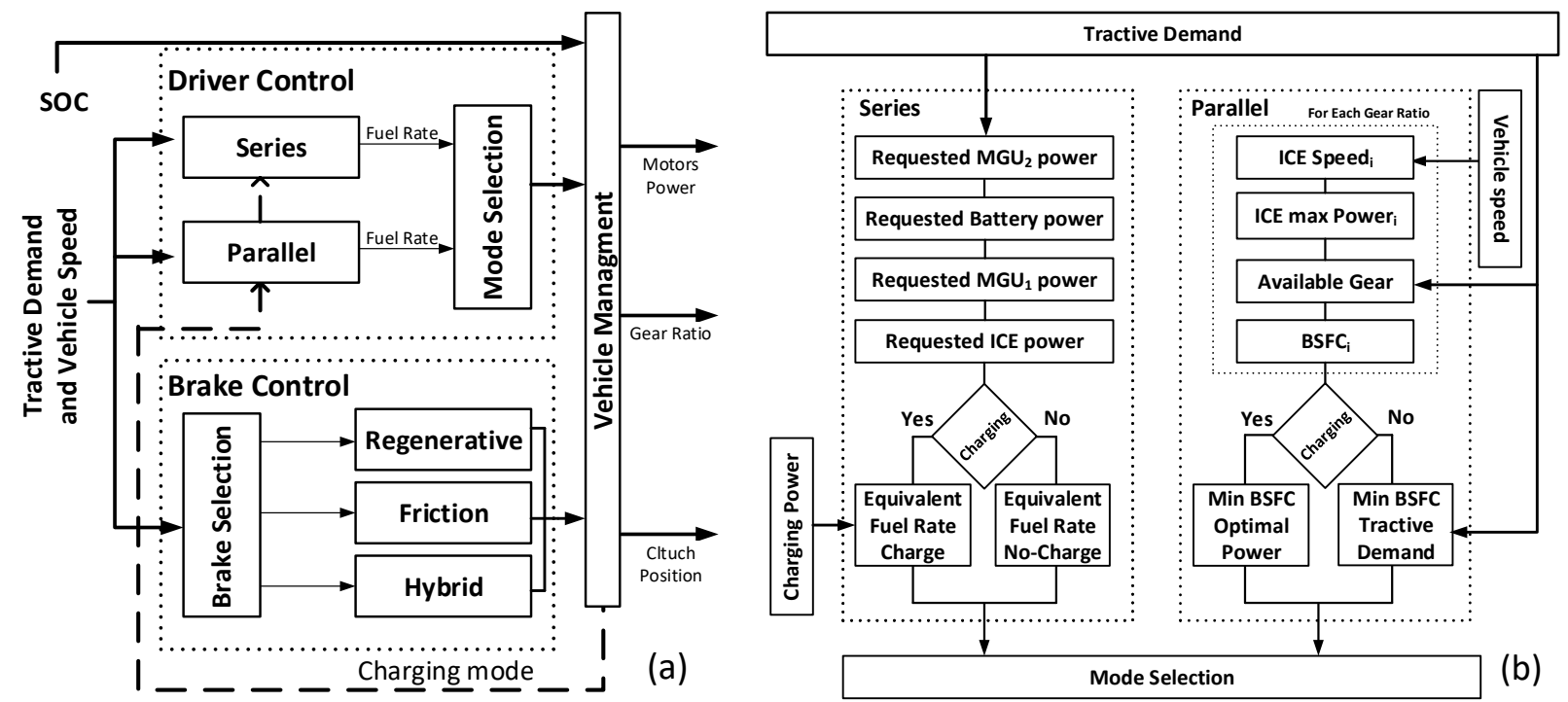

Fig. 2. Flowchart of the EFRMS (a) and of procedure for the EFR calculation (b)
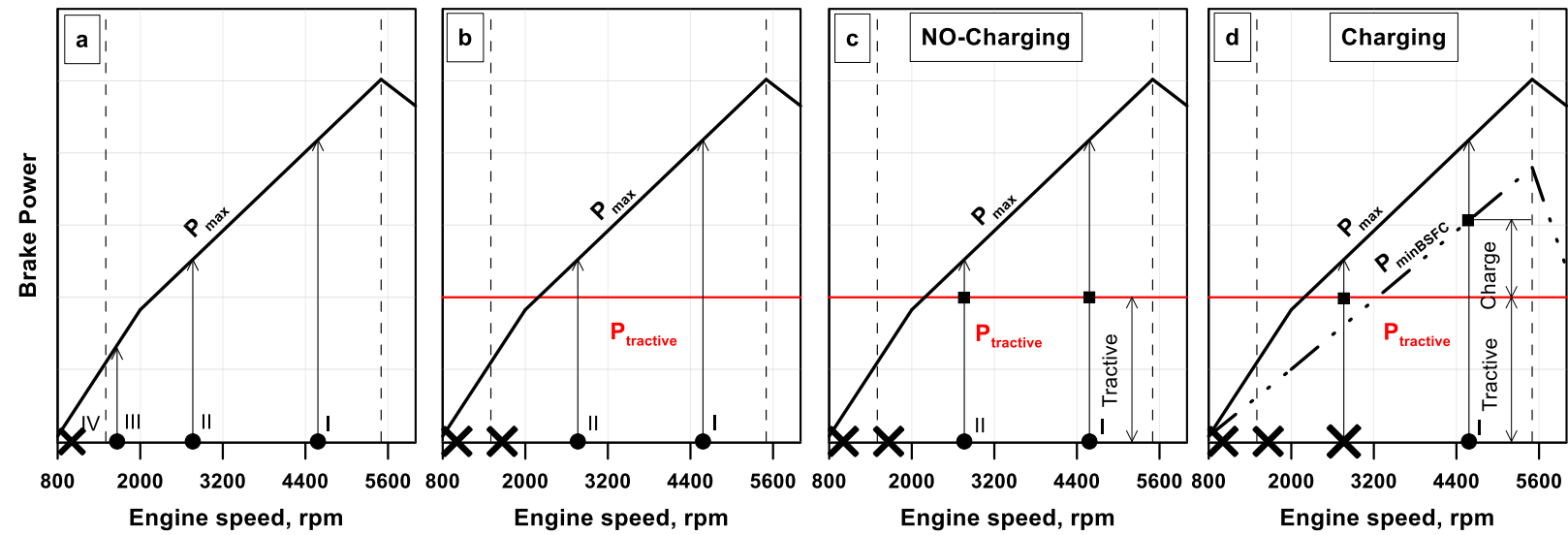

Fig. 3. Main steps of the gear ratio selection of the EFRMS in parallel mode

For the remaining gear ratios, the rated ICE power and the related BSFC can be hence calculated, along with the corresponding fuel rates. To this purpose, different methodologies are applied depending on the charging state. In nocharging condition (Fig. 3c), the ICE is assumed to fully supply the tractive demand $\left(P_{\text {tractive }}\right.$ in Fig. 3c). Instead, in charging condition (Fig. 3d), the ICE delivers the power of minimum BSFC, which is split on one side to satisfy the tractive demand and on the other to charge the battery. If the ICE, working in minimum BSFC condition, is not able to satisfy the tractive demand, the corresponding gear number is discarded (the second gear ratio in the example of 
Fig. 3d). Among the gear ratios which pass the above tests, the one providing the minimum fuel rate is selected to define the $\mathrm{EFR}_{\text {parallel. }}$ A tunable penalization term is added to the $\mathrm{EFR}_{\text {parallel }}$ for the gear ratios different from the current one to avoid excessive gearshifts. In the parallel mode, the power requested to the ICE is computed once again starting from the vehicle power demand by the wheels. In this case, differently from the series mode, only the losses in the gearbox $_{1}$ have to be considered along the energy path between the wheels and the ICE. As a final remark, in the parallel mode, if the ICE is not able to satisfy the tractive demand, the vehicle driving is supported by the $\mathrm{MGU}_{2}$. It is worth to underline that the proposed control strategy is versatile and suitable to any hybrid vehicle architecture. The application here described represents just an example for a quite complex test case.

\section{Results discussion}

The vehicle management strategies, described in the previous section, are applied for the simulation of a WLTC for a segment $\mathrm{C}$ vehicle with different operating conditions in terms of auxiliary consumption, car mass and BSFC map, summing up 10 cases (Table 1). Before discussing the $\mathrm{CO}_{2}$ emission assessment between the PMP and the EFRMS, some detailed comparisons for a single test case (\# 6 of Table 1), about the powertrain and battery management differences, will be presented.

Table 1. Test matrix

\begin{tabular}{lllllllllll}
\hline Case \# & $\# 1$ & $\# 2$ & $\# 3$ & $\# 4$ & $\# 5$ & $\# 6$ & $\# 7$ & $\# 8$ & $\# 9$ & $\# 10$ \\
\hline Vehicle mass $(\mathrm{kg})$ & 1659 & 1679 & 1759 & 1759 & 1759 & 1659 & 1679 & 1759 & 1759 & 1759 \\
Auxiliary consumption $(\mathrm{kW})$ & 0.40 & 0.40 & 0.40 & 0.75 & 0.00 & 0.40 & 0.40 & 0.40 & 0.00 & 075 \\
$\mathrm{BSFC}_{\text {min }}(\mathrm{g} / \mathrm{kWh})$ & $\mathrm{BSFC}_{1}$ & $\mathrm{BSFC}_{1}$ & $\mathrm{BSFC}_{1}$ & $\mathrm{BSFC}_{1}$ & $\mathrm{BSFC}_{1}$ & $\mathrm{BSFC}_{2}$ & $\mathrm{BSFC}_{2}$ & $\mathrm{BSFC}_{2}$ & $\mathrm{BSFC}_{2}$ & $\mathrm{BSFC}_{2}$ \\
\hline
\end{tabular}
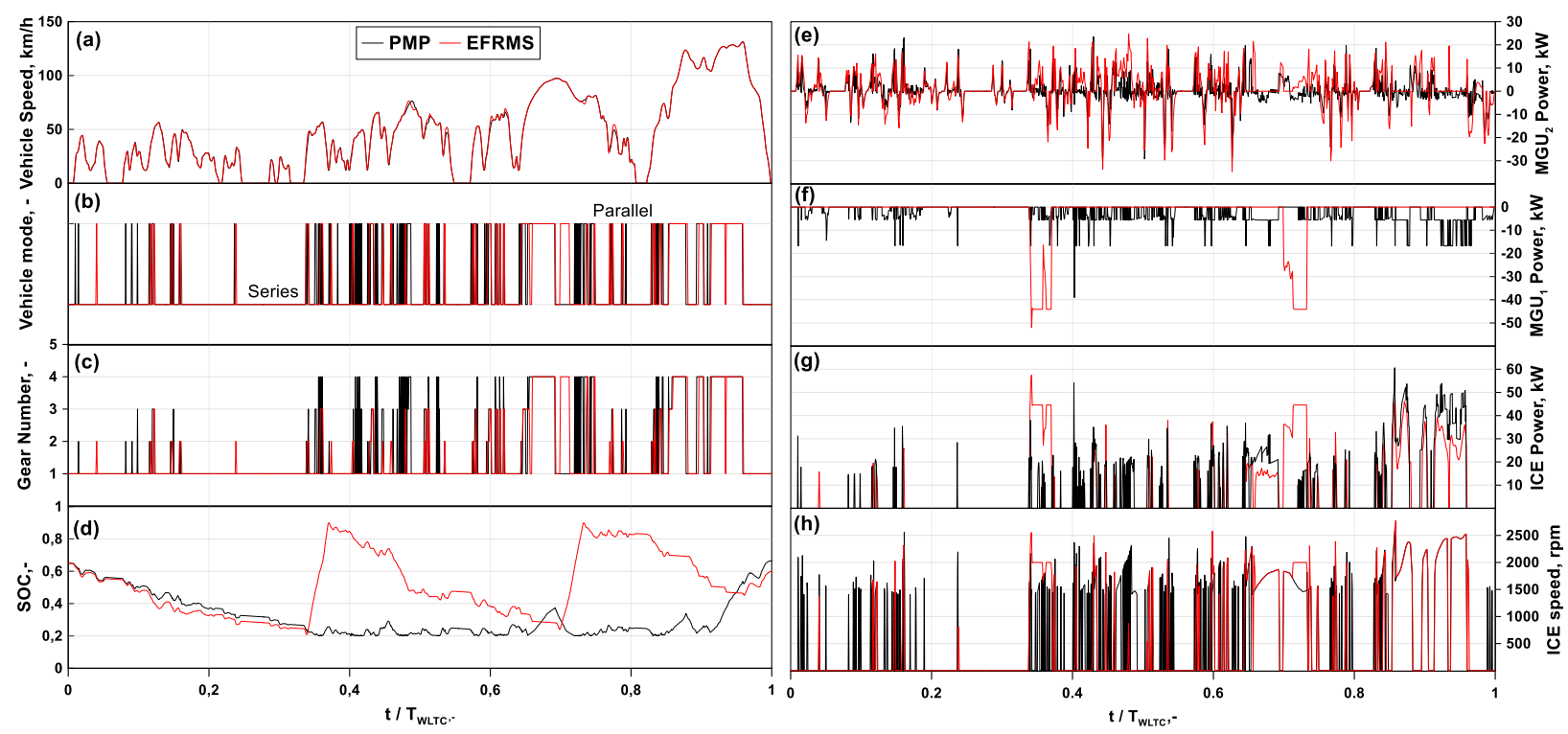

Fig. 4. PMP/EFRMS comparison of vehicle speed (a), vehicle mode (b), gear number (c), SOC (d), $\mathrm{MGU}_{2}$ power (e), $\mathrm{MGU}_{1}$ power (f), ICE power (g) and ICE speed (h) along a WLTC.

To this aim the vehicle speed profile, the vehicle mode (series/parallel), the selected gear number of the gear-box 2 , and the battery SOC are compared on the left side of Fig. 4 and plotted against the normalized time. The power delivered/absorbed by the electric and the thermal units, in addition to the ICE speed, are analyzed on the right side of Fig. 4 (a positive value indicates that the power is delivered by the unit). As a first remark, it can be noted that the vehicle WLTC speed profile is matched by both PMP and EFRMS (Fig. 4a), but the control of the powertrain components is frequently different. Starting from the mode profile (Fig. 4b), it can be observed that the series (parallel) 
mode is preferred at the beginning (end) of the cycle for both EMSs. Some differences arise in the cycle middle, where PMP privileges the parallel mode, while the EFRMS more frequently choses the series one.

The series/parallel selection also reflects on the gear number profile of the gearbox 2 , shown in Fig. 4c. Indeed, when the parallel mode is chosen by both PMP and EFRMS, the gear number is very often the same. This is consistent with the advance to operate the thermal unit in the conditions of minimum fuel consumption (speeds between 1500 $\mathrm{rpm}$ and $2500 \mathrm{rpm}$ for the considered engine, as shown in Fig. 4h). The mode and gear number comparisons (Fig. 4b and Fig. 4c) also underline that PMP involves more frequent changes, which are due to lower penalizations of the events of series/parallel switches and gearshifts. The SOC assessment in Fig. $4 \mathrm{~d}$ and requested $\mathrm{MGU}_{2}$ power (Fig. 4e) put into evidence that, during the initial phase of the cycle (up to about $0.35 \mathrm{t} / \mathrm{T}_{\mathrm{WLTC}}$ ), both the methodologies prefer almost an electric driving, resulting in similar battery discharging profiles. When the SOC reaches the lower threshold, the EFRMS activates the charging mode. This last appears as a sudden SOC increase, which ends when the SOC achieves the upper threshold. The charging modality also affects the gearbox control, resulting in a downshift (Fig. $4 \mathrm{c}$ ) and in a sudden increase of the engine power (Fig. 4g). Both those actions, as said in the previous section, have the aim of making the engine to operate with the minimum possible fuel consumption. Once charged the battery, in the cycle middle, the vehicle demand is fulfilled by the $\mathrm{MGU}_{2}$, while the power peaks are supplied by the ICE. In those cases, the series mode is activated once again in a privilege, and only occasionally the parallel one is enabled. Concerning the PMP approach, in the middle stage of the cycle, the SOC level remains close to the minimum threshold, and the parallel strategy is more frequently preferred compared to the EFRMS case. In this phase, the power delivered by the ICE is split and employed to move the vehicle and to charge the battery. Moving to the ending highspeed cycle section (after about $0.65 \mathrm{t} / \mathrm{T}_{\text {WLTC }}$ ), the strategies go back to being very similar, in terms of gear ratio and power delivered by the thermal unit. In this phase, the vehicle power demand is primarily supplied by the ICE, while the $\mathrm{MGU}_{2}$ is used to fulfill the power request peaks and for the regenerative breakings. Indeed, both the strategies promote the parallel mode since, at the high speed and load, the ICE works near its minimum BSFC. In addition, as put in evidence in Fig. 4h, the ICE is turned off to maximize the recoverable energy during the deceleration without resort to the engine braking.
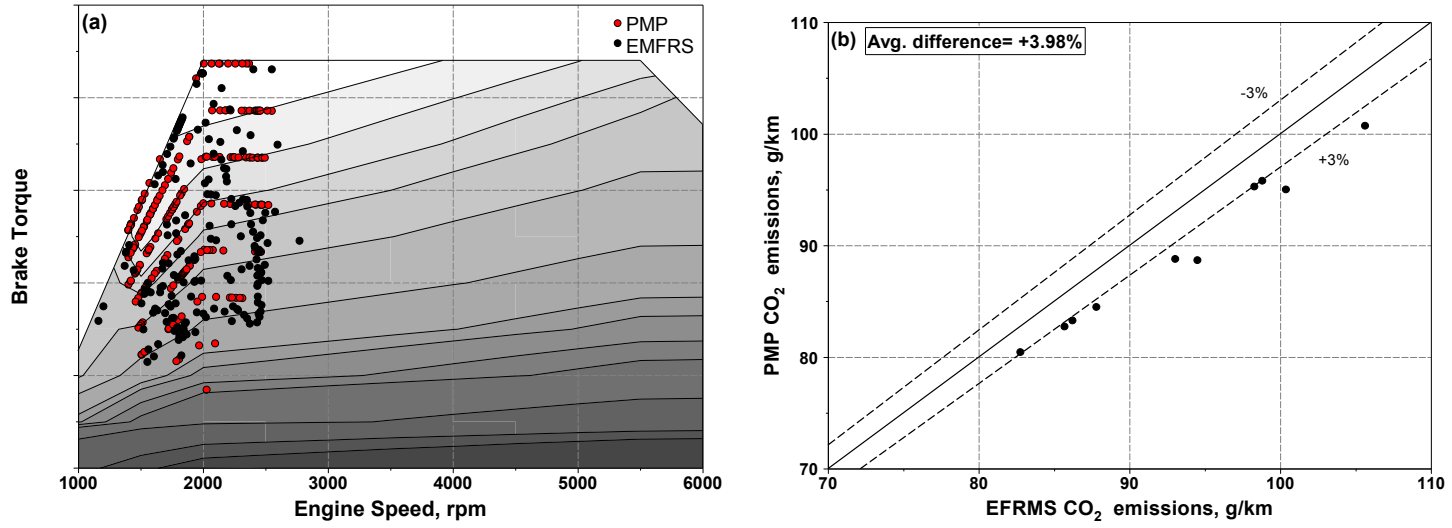

Fig. 5. PMP/EFRMS comparison of engine experienced operating points (a) and $\mathrm{CO}_{2}$ production (b) along a WLTC.

Main differences concern the charging strategies, as put into evidence by the SOC profile. The PMP goes ahead in maintaining a reduced SOC level, very close to the lower threshold, till the ending stage of the cycle (after about 0.90 $\mathrm{t} / \mathrm{T}_{\text {WLTC }}$ ). Taking advantage of the regenerative breaking at the cycle end, the battery is charged in order to have a final SOC close to the initial state. The battery charge is also provided by a certain contribution from the ICE, as highlighted by the $\mathrm{MGU}_{1}$ power profile (Fig. 4f). Of course, the viability of this strategy is strictly related to the "a-priori" knowledge of the whole driving cycle, which is characteristic of a GOS approach such as the PMP. Conversely, the EFRMS involves a second charging phase, at the beginning of the high-speed cycle stage (about at $0.70 \mathrm{t} / \mathrm{T}_{\text {WLTC }}$ ), where once again the SOC reaches the upper limit. The subsequent SOC reduction is due to both the auxiliary and $\mathrm{MGU}_{2}$ absorptions. Despite the above discussed differences in the thermal unit management, the conditions experienced by the ICE lie in the same portion of the operating map, as underlined in Fig. 5a. The latter also shows the BSFC map under the form of grayscale. Both control strategies make the engine to operate in a speed range 
between $1500 \mathrm{rpm}$ and $2500 \mathrm{rpm}$, and with a load as high as possible, which corresponds to the minimum BSFC area. As expected, to avoid inefficient operation, the ICE never works at very low load. When the power demand required by the vehicle/battery is reduced, a pure electric driving is hence preferred.

The global effectiveness of the PMP and EFRMS strategies are finally assessed in terms of $\mathrm{CO}_{2}$ emissions along the WLTC, as shown in Fig. 5b. This comparison underlines that both strategies respond in a very similar way to the variations of the vehicle mass, auxiliary consumption and engine efficiency. As expected, the PMP approach foresees lower $\mathrm{CO}_{2}$ emissions, with levels 3-4\% minor than the EFRMS results. This is mainly due to the possibility of the PMP to better control the battery charge/discharge strategy. As a final consideration, the proposed EFRMS approach has demonstrated an adequate reliability under various vehicle configurations, with an acceptable $\mathrm{CO}_{2}$ penalization compared to the "theoretical" PMP target.

\section{Conclusion}

In this work, an innovative LOS for series/parallel HEV, labelled as EFRMS, was presented in detail and compared to a well-assessed global optimization strategy, based on the PMP. The numerical study regarded a HEV of the segment $\mathrm{C}$, which is representative of the European market. First, the considered engine was schematized in a lumpedparameter model. The thermal unit was characterized by its BSFC map, while the other mechanical/electrical components were assumed to have a constant efficiency. The strategies were compared in terms of $\mathrm{CO}_{2}$ emissions along a WLTC. Various vehicle/powertrain architectures were investigated, holding different vehicle mass, auxiliary consumption and ICE efficiency.

The results highlighted that the EFRMS strategy determined a powertrain management similar to the PMP one during the urban driving, where an almost pure electric/series driving was applied. The same occurred during the highway portion of the driving cycle, where the thermal unit was preferred to fulfill the vehicle power demand. The main differences between the strategies concerned the battery charging method. Because of the above differences, the EFRMS determined higher $\mathrm{CO}_{2}$ emissions (about 3-4 \%) along the driving cycle compared to the PMP results. The EFRMS-related $\mathrm{CO}_{2}$ penalization appeared acceptable in the light of the absence of "a-priori" information about the driving cycle. The proposed methodology could represent an effective path to improve the $\mathrm{CO}_{2}$ emissions in an actual "on-vehicle" application.

\section{Acknowledgement}

"This project has received funding from the European Union's Horizon 2020 research and innovation programme under grant agreement No 724084".

\section{References}

[1] Morita K. (2003). Automotive power source in 21st century. JSAE Review, 24(1), 3-7.

[2] Guzzella L., \& Sciarretta A. (2005). Vehicle propulsion systems: introduction to modeling and optimization. Springer Verlag.

[3] Serrao L., Onori S., Rizzoni G. (2011). A comparative analysis of energy management strategies for hybrid electric vehicles. Journal of Dynamic Systems, Measurement, and Control, 133(3), 031012 1-9.

[4] Salmasi F. R. (2007). Control Strategies for Hybrid Electric Vehicles: Evolution, Classification, Comparison, and Future Trends. IEEE Transactions on Vehicular Technology, 56(5), 2393-2404.

[5] Chasse, A., \& Sciarretta, A. (2011). Supervisory control of hybrid powertrains: An experimental benchmark of offline optimization and online energy management. Control Engineering Practice, 19(11), 1253-1265.

[6] Pérez L. V., Bossio G. R., Moitre D., \& García G. O. (2006). Optimization of power management in a hybrid electric vehicle using Dynamic Programming. Mathematics and Computers in Simulation, 73(1-4), 244-254.

[7] Paganelli G., Ercole G., Brahma A., Guezennec Y., \& Rizzoni G. (2001). General supervisory control policy for the energy optimization of charge-sustaining hybrid electric vehicles. JSAE review, 22(4), 511-518.

[8] Lin C., Peng H., \& Grizzle J. (2004). A stochastic control strategy for hybrid electric vehicles. American Control Conference. $4710-4715$.

[9] European Environment Agency, “Annual European Union greenhouse gas inventory 1990-2012 and inventory report 2014, Report No.: 9/2014", 2014

[10] Kim, N., Cha, S., \& Peng, H. (2011). Optimal control of hybrid electric vehicles based on Pontryagin's minimum principle. IEEE Transactions on Control Systems Technology, 19(5), 1279-1287. 